Article

\title{
Integral Representations for Products of Two Bessel or Modified Bessel Functions
}

\author{
Dragana Jankov Maširević ${ }^{1}$ (D) and Tibor K. Pogány ${ }^{2,3, * \mathbb{D}}$ \\ 1 Department of Mathematics, University of Osijek, Trg Lj. Gaja 6, 31000 Osijek, Croatia; djankov@mathos.hr \\ 2 Faculty of Maritime Studies, University of Rijeka, Studentska 2, 51000 Rijeka, Croatia \\ 3 Institute of Applied Mathematics, Óbuda University, Bécsi út 96/b, 1034 Budapest, Hungary \\ * Correspondence: poganj@pfri.hr
}

Received: 4 September 2019; Accepted: 14 October 2019; Published: 16 October 2019

check for updates

\begin{abstract}
The first part of the article contains integral expressions for products of two Bessel functions of the first kind having either different integer orders or different arguments. A similar question for a product of modified Bessel functions of the first kind is solved next, when the input functions are of different integer orders and have different arguments.
\end{abstract}

Keywords: Bessel function of the first kind; modified Bessel function of the first kind; integral representation of product functions; Chebyshev polynomial of the first kind

MSC: Primary: 33C10; Secondary: 33C05; 33C20

\section{Introduction and Motivation}

Integral representations of products of two Bessel functions and/or modified Bessel functions have been studied over the years by several authors. For example, consider Schläfli's formula (for integer order Bessel functions of the first kind $\left.J_{v}(z), v \in \mathbb{Z}\right)([1]$, p. 142)

$$
J_{\mu}(z) J_{v}(z)=\frac{2}{\pi} \int_{0}^{\pi / 2} J_{\mu+v}(2 z \cos t) \cos ((\mu-v) t) \mathrm{d} t, \quad \Re(\mu \pm v) \in \mathbb{Z},
$$

whose validity for general $\mu, v$ has been shown by Gegenbauer ([2], p. 567) (also consult ([3], pp. 150-151, §5.43)). Dixon and Ferrar stated several formulae for such a product by scaling the arguments ([4], p. 206, Equation (7.22))

$$
J_{\mu}(X z) J_{v}(x z)=\frac{X^{\mu} x^{v}}{\pi} \int_{-\pi / 2}^{\pi / 2} \mathrm{e}^{\mathrm{i}(\mu-v) t}\left(\lambda_{1} / \lambda_{2}\right)^{\mu+v} J_{\mu+v}\left(z \lambda_{1} \lambda_{2}\right) \mathrm{d} t,
$$

where $\lambda_{1}^{2}=2 \cos t, \lambda_{2}=\sqrt{X^{2} \mathrm{e}^{\mathrm{it}}+x^{2} \mathrm{e}^{-\mathrm{it} t}}$, while Nicholson proved that ([5], p. 236, Equation (39))

$$
J_{n}(z) J_{-n}(z)=\frac{1}{\pi \sin (n \pi)} \int_{0}^{\pi} \sin (2 n x) J_{0}(2 z \sin x) \mathrm{d} x,
$$

where $z \in \mathbb{R}$ and $n \notin \mathbb{Z}$. This result implies for $n$ non-negative integer the formula ([5], p. 236, Equation (40))

$$
J_{n}^{2}(z)=\frac{1}{\pi} \int_{0}^{\pi} \sin (2 n x) J_{0}(2 z \sin x) \mathrm{d} x .
$$

Nicholson also derived integral representation for the mixed product $J_{n}(z) Y_{n}(z)$ of the Bessel function of the first kind $J_{n}(z)$ and of the Bessel function of the second kind $Y_{n}(z)$ when $n \in \mathbb{Z}$; similar results can be found also in the already cited papers by Dixon and Ferrar [4,6] and by Görlich et al. [7]. 
Glasser and Montaldi ([8], p. 578, Equation (2.1)) started with the expansion (derived from the series definition of $J_{v}(z), z, v \in \mathbb{C}$ ) which seemingly originates back to Watson ([3], p. 148, Equation (2))

$$
J_{\mu}(a x) J_{v}(b x)=\frac{a^{\mu} b^{v} x^{\mu+v}}{2^{\mu+v} \Gamma(\mu+1) \Gamma(v+1)} \sum_{m \geq 0} \frac{(-1)^{m}(a x)^{2 m}}{4^{m}(\mu+1)_{m} m !}{ }_{2} F_{1}\left[\begin{array}{c}
-m,-\mu-m \\
v+1
\end{array} \mid \frac{b^{2}}{a^{2}}\right],
$$

where $\min \{\Re(v), \Re(\mu)\}>-1,|\Re(b / a)|<1$ and $x \in \mathbb{C}$, in order to derive the integral expression in terms of the modified Bessel functions of the first kind $I_{\nu}$ of the order $v$ and of the second kind $K_{\mu}$ of the order $\mu$ in the integrand ([8], p. 578, Equation (2.4))

$$
\begin{aligned}
J_{\mu}(a x) J_{\nu}(b x)= & \frac{4}{\Gamma(\mu+1) \Gamma(v+1) \Gamma(\mu+v+1)}\left(\frac{a x}{2}\right)^{\mu}\left(\frac{b x}{2}\right)^{v}\left(1-\frac{b^{2}}{a^{2}}\right)^{\mu+v+1}\left(\frac{a}{b}\right)^{v} \\
& \times \int_{0}^{\infty} t^{\mu+v+1}{ }_{0} F_{3}\left[\mu+1, v+1, \mu+v+1 \mid-z^{2} t^{2}\right] K_{\mu}(2 t) I_{\nu}\left(\frac{2 b t}{a}\right) \mathrm{d} t,
\end{aligned}
$$

where $\min \{\Re(v), \Re(\mu+v)\}>-1$ and $|\Re(b / a)|<1$ with the shorthand $z=a x\left(1-b^{2} / a^{2}\right) / 2$.

Finally, Hrycak and Schmutzhard ([9], p. 171) studied an integral representation for a product of two modified Bessel functions of the second kind $K_{v} K_{\mu}$ and established integral representation for the cross-product of the Bessel functions $J_{\nu}$ and $Y_{\mu}$, which is a generalization of Nicholson's integral for $J_{v}^{2}(x)+Y_{v}^{2}(x)$, see ([6], p. 137 et seq.) and also consult the ancestor result ([5], p. 234, Equation (34)).

It is important to mention that all the formulae valid for Bessel functions of the first kind $J_{v}$ are also valid for the modified Bessel functions of the first kind $I_{v}$, knowing the identity $I_{v}(x)=\mathrm{i}^{-v} J_{v}(\mathrm{i} x)$ and its reversed counterpart formula.

The motivation for writing this article concerning integral form expressions of a product of two Bessel (or modified Bessel) functions of the first kind with scaled arguments is manifold. Firstly, we intend to complement with new inputs the existing integral representations lists for a products of two in specialized manuals of Bessel functions and/or directories on special functions, compare [10-15] together with a few volumes of relevant Prudnikov-Brychkov-Marichev monographs; secondly, we prepare novel integral form tools for establishing functional or uniform bounding inequalities for the considered products of Bessel functions (this goal heavily overgrows the purposes and tasks of the recent article, so, we leave it for another address); thirdly, we explore possible new applications not only in topics and problems where Neumann, Kapteyn, Schlömilch or Dini series of products of Bessel functions occur either in theoretical or modeling application items (see [16] and the appropriate exhaustive references list therein), but also for instance in harmonic analysis [17] or representation theory [18]. Lastly, we even found this project problem of interest to study per se by using certain classical analysis tools like series multiplication and some technical actions such as replacing the order of summation and integration, integral transformations for special functions of Bessel and/or hypergeometric type.

The structure of the article is as follows. Firstly, we obtain certain expressions for the products of two Bessel functions of the first kind of different orders or different arguments relying on Equation (2) presented in the next section. The Section 3 consists of our main result; there we expose a general integral representation for the product of two modified Bessel functions of the first kind, with integer orders constrained as $\mu-v=n \in \mathbb{N}$ and also for different arguments. We close this section with the main formula when $\mu \pm v \in\{1,2,3\}$. Finally, in the "Discussion" section we connect the results from the Section 2 (Theorems 1 and 2) devoted to the products of two Bessel functions with the integral expressions inferred for the product of two modified Bessel functions in Theorem 3. We finish the exposition with further suggested and posed research directions. 


\section{Integral Representation of $J_{v}(a x) J_{\mu}(b x)$}

Our first result concerns an integral form of the product $J_{\nu}(x) J_{\mu}(x)$ when the relation of orders is not restricted. While the results immediately follow from the already known formulae mentioned below in the proof, we present them for the sake of completeness of exposition.

Theorem 1. For all $x \in \mathbb{C}$ we have

$$
\begin{aligned}
& J_{\mu}(x) J_{\nu}(x)=\frac{x^{\mu+v}}{2^{\mu+v} \Gamma(\mu+1) \Gamma(v+1) \mathrm{B}\left(\frac{v+\mu}{2}+1, \frac{v+\mu}{2}\right)} \\
& \times \int_{0}^{1} t^{(v+\mu) / 2}(1-t)^{(v+\mu) / 2-1}{ }_{1} F_{2}\left[\begin{array}{c}
\frac{v+\mu+1}{2} \\
v+1, \mu+1
\end{array} \mid-x^{2} t\right] \mathrm{d} t, \quad \Re(v+\mu)>0 \\
& =\frac{x^{\mu+v}}{2^{\mu+v} \Gamma(\mu+1) \Gamma(v+1) \Gamma\left(\frac{v+\mu}{2}+1\right)} \\
& \times \int_{0}^{\infty} \mathrm{e}^{-t} t^{(v+\mu) / 2}{ }_{1} F_{3}\left[\begin{array}{c|l}
\frac{v+\mu+1}{2} \\
v+1, \mu+1, v+\mu+1
\end{array} \mid-x^{2} t\right] \mathrm{d} t, \quad \Re(v+\mu)>-1 ;
\end{aligned}
$$

where in Equation (4) we constrain $\Re\left(x^{2}\right)>-1$, while $\mathrm{B}(s, t), \min \{\Re(s), \Re(t)\}>0$ stands for the Beta function.

Proof. Consider formula ([19], p. 216, Equation (39))

$$
J_{\mu}(x) J_{v}(x)=\frac{x^{\mu+v}}{2^{\mu+v} \Gamma(\mu+1) \Gamma(v+1)}{ }_{2} F_{3}\left[\begin{array}{c}
\frac{v+\mu+1}{2}, \frac{v+\mu}{2}+1 \\
v+1, \mu+1, v+\mu+1
\end{array} \mid-x^{2}\right], \quad \Re(v+\mu)>-1 .
$$

Recalling two integral representation formulae for the generalized hypergeometric function ([15], p. 408, Equations (16.5.2)-(16.5.3))

$$
\begin{aligned}
{ }_{2} F_{3}\left[\begin{array}{c}
a_{1}, a_{2} \\
b_{1}, b_{2}, b_{3}
\end{array} \mid z\right] & =\frac{\Gamma\left(b_{1}\right)}{\Gamma\left(a_{1}\right) \Gamma\left(b_{1}-a_{1}\right)} \int_{0}^{1} t^{a_{1}-1}(1-t)^{b_{1}-a_{1}-1}{ }_{1} F_{2}\left[\begin{array}{c}
a_{2} \\
b_{2}, b_{3}
\end{array} \mid z t\right] \mathrm{d} t \\
& =\frac{1}{\Gamma\left(a_{1}\right)} \int_{0}^{\infty} \mathrm{e}^{-t} t^{a_{1}-1}{ }_{1} F_{3}\left[\begin{array}{c}
a_{2} \\
b_{1}, b_{2}, b_{3}
\end{array} \mid z t\right] \mathrm{d} t
\end{aligned}
$$

provided $\Re\left(b_{1}\right)>\Re\left(a_{1}\right)>0$ in the previous, while $\Re\left(a_{1}\right)>0, \Re(z)<1$ in the latter case, we deduce the asserted relations.

Bearing in mind the connecting relation $J_{v}(i x)=i^{v} I_{v}(x)$ we immediately conclude

Corollary 1. For all $x \in \mathbb{C}$ we have

$$
\begin{aligned}
& I_{\mu}(x) I_{v}(x)= \frac{x^{\mu+v}}{2^{\mu+v} \Gamma(\mu+1) \Gamma(v+1) \mathrm{B}\left(\frac{v+\mu}{2}+1, \frac{v+\mu}{2}\right)} \\
& \times \int_{0}^{1} t^{(v+\mu) / 2}(1-t)^{(v+\mu) / 2-1}{ }_{1} F_{2}\left[\begin{array}{c}
\frac{v+\mu+1}{2} \\
v+1, \mu+1
\end{array} \mid x^{2} t\right] \mathrm{d} t, \quad \Re(v+\mu)>0 \\
&= \frac{x^{\mu+v}}{2^{\mu+v} \Gamma(\mu+1) \Gamma(v+1) \Gamma\left(\frac{v+\mu}{2}+1\right)} \\
& \quad \times \int_{0}^{\infty} \mathrm{e}^{-t} t^{(v+\mu) / 2}{ }_{1} F_{3}[v+1, \mu+1, v+\mu+1 \\
&\left.v+x^{2} t\right] \mathrm{d} t, \quad \Re(v+\mu)>-1,
\end{aligned}
$$

provided $\Re\left(x^{2}\right)<1$ in the latter integral. 
Moreover, we consider the case when the input Bessel functions possess scaled arguments but their orders coincide.

Theorem 2. For all $x \in \mathbb{C}, \Re(v)>-1 / 2$ and $a, b \in \mathbb{R}$ such that $|a| \geq|b|$ there holds

$$
J_{v}(a x) J_{v}(b x)=\frac{(a b)^{v} x^{v}}{2^{v} \sqrt{\pi} \Gamma\left(v+\frac{1}{2}\right)} \int_{-1}^{1} \frac{\left(1-u^{2}\right)^{v-1 / 2}}{\left(a^{2}+b^{2}+2|a b| u\right)^{v / 2}} J_{v}\left(x \sqrt{a^{2}+b^{2}+2|a b| u}\right) \mathrm{d} u .
$$

Proof. Let us introduce the ultraspherical or Gegenbauer function ([20], §3.15.2)

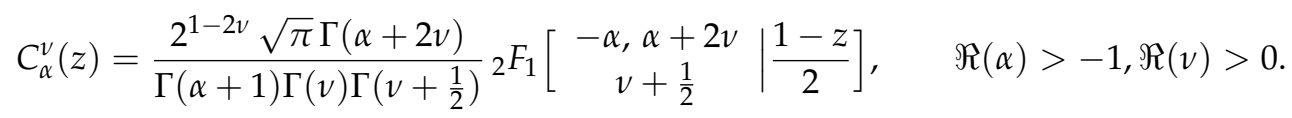

A Gauss-Kummer quadratic transform of Gauss hypergeometric function ([15], p. 391, Equation (15.8.15)) reads

$$
{ }_{2} F_{1}\left[\begin{array}{c|c}
c, d \\
c-d+1
\end{array} \mid z\right]=(1+z)^{-c}{ }_{2} F_{1}\left[\begin{array}{c}
\frac{c}{2}, \frac{c+1}{2} \\
c-d+1
\end{array} \mid \frac{4 z}{(1+z)^{2}}\right], \quad z \in(-1,1],
$$

provided both hypergeometric functions exist. This relation implies that

$$
{ }_{2} F_{1}\left[\begin{array}{c|c}
-m,-v-m \\
v+1
\end{array} \mid \frac{b^{2}}{a^{2}}\right]=\left(1+\frac{b^{2}}{a^{2}}\right)^{m}{ }_{2} F_{1}\left[\begin{array}{c}
-\frac{m}{2}, \frac{1-m}{2} \\
v+1
\end{array} \mid\left(\frac{2 a b}{a^{2}+b^{2}}\right)^{2}\right],
$$

under the condition $|b| \leq|a|$. Moreover, employing the identity [21]

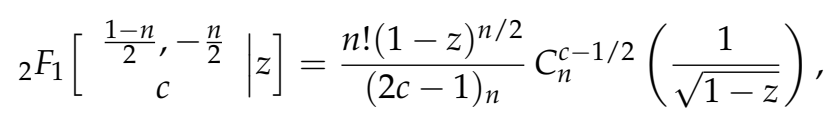

Equation (6) becomes

$$
{ }_{2} F_{1}\left[\begin{array}{c|c}
-m,-v-m \\
v+1
\end{array} \mid \frac{b^{2}}{a^{2}}\right]=\left(1-\frac{b^{2}}{a^{2}}\right)^{m} \frac{m !}{(2 v+1)_{m}} C_{m}^{v+1 / 2}\left(\frac{a^{2}+b^{2}}{a^{2}-b^{2}}\right) .
$$

Rewriting the multiplication constant in the integral ([20], p. 178, Equation (22)) suitable for our purposes, we get the expression

$$
C_{\alpha}^{v}(z)=\frac{2^{1-2 v} \Gamma(\alpha+2 v)}{\Gamma(\alpha+1) \Gamma^{2}(v)} \int_{0}^{\pi}\left(z+\sqrt{z^{2}-1} \cos t\right)^{\alpha} \sin ^{2 v-1} t \mathrm{~d} t, \quad \Re(v)>0 .
$$

Then, there holds

$$
C_{m}^{v+1 / 2}\left(\frac{a^{2}+b^{2}}{a^{2}-b^{2}}\right)=\frac{(2 v+1)_{m} \Gamma(2 v+1)}{4^{v} m ! \Gamma^{2}\left(v+\frac{1}{2}\right)} \int_{0}^{\pi}\left(\frac{a^{2}+b^{2}}{a^{2}-b^{2}}+\frac{2|a b|}{a^{2}-b^{2}} \cos t\right)^{m} \sin ^{2 v} t \mathrm{~d} t,
$$

or equivalently, from Equation (2) follows

$$
\begin{gathered}
J_{v}(a x) J_{v}(b x)=\frac{\left(a b x^{2}\right)^{v}}{\sqrt{\pi} 4^{v} \Gamma(v+1) \Gamma\left(v+\frac{1}{2}\right)} \int_{0}^{\pi} \sin ^{2 v} t \sum_{m \geq 0} \frac{(-1)^{m}\left[x^{2}\left(a^{2}+b^{2}+2|a b| \cos t\right)\right]^{m}}{4^{m}(v+1)_{m} m !} \mathrm{d} t \\
=\frac{\left(a b x^{2}\right)^{v}}{\sqrt{\pi} 4^{v} \Gamma(v+1) \Gamma\left(v+\frac{1}{2}\right)} \int_{0}^{\pi} \sin ^{2 v} t \cdot{ }_{0} F_{1}\left[\begin{array}{c}
- \\
v+1
\end{array} \mid-\frac{x^{2}}{4}\left(a^{2}+b^{2}+2|a b| \cos t\right)\right] \mathrm{d} t
\end{gathered}
$$




$$
=\frac{(a b x)^{v}}{\sqrt{\pi} 2^{v} \Gamma\left(v+\frac{1}{2}\right)} \int_{0}^{\pi} \frac{\sin ^{2 v} t}{\left(a^{2}+b^{2}+2|a b| \cos t\right)^{v / 2}} J_{v}\left(x \sqrt{a^{2}+b^{2}+2|a b| \cos t}\right) \mathrm{d} t,
$$

where in the last equality we applied the connection formula

$$
{ }_{0} F_{1}\left[\begin{array}{c}
- \\
b
\end{array} \mid z\right]=\Gamma(b)(-z)^{\frac{1-b}{2}} J_{b-1}(2 \sqrt{-z}) \text {. }
$$

Finally, substitution of $\cos t=u$ and the transformation $\sin (\arccos (z))=\sqrt{1-z^{2}}$ yields the statement.

The 'modified' counterpart result to Theorem 2 follows from Equation (5) inserting i $x$ instead of $x$ for arguments on both sides.

Corollary 2. For all $x \in \mathbb{C}, \Re(v)>-1 / 2$ and $a, b \in \mathbb{R}$ such that $|a| \geq|b|$ there holds

$$
I_{v}(a x) I_{v}(b x)=\frac{(a b)^{v} x^{v}}{2^{v} \sqrt{\pi} \Gamma\left(v+\frac{1}{2}\right)} \int_{-1}^{1} \frac{\left(1-u^{2}\right)^{v-1 / 2}}{\left(a^{2}+b^{2}+2|a b| u\right)^{v / 2}} I_{v}\left(x \sqrt{a^{2}+b^{2}+2|a b| u}\right) \mathrm{d} u .
$$

\section{Integral Representation of $I_{\mu} I_{\nu}$}

The history of the integral expressions for the product of two modified Bessel functions of the first kind $I_{\mu} I_{\nu}$ is well-covered for instance in Watson's monograph ([3] (\$13.72-73)). More precisely, the counterpart formula to Equation (1) reads ([3], p. 441, Equation (2))

$$
I_{\mu}(z) I_{v}(z)=\frac{2}{\pi} \int_{0}^{\frac{\pi}{2}} I_{\mu+v}(2 z \cos \vartheta) \cos ((\mu-v) \vartheta) \mathrm{d} \vartheta, \quad \Re(\mu+v)>-1 .
$$

We derive now another expression for $I_{\mu} I_{\nu}$ when the integer orders $\mu, v$ of building functions are constrained as $\mu-v=n \in \mathbb{N}$, say; because of the parity property $I_{-v}(z)=I_{v}(z), v \in \mathbb{Z}$ we can reduce our consideration to positive integers $n$ without losing generality. For that purpose we begin with the approach suggested by Watson, concerning the proof of the Nicholson formula ([3], p. 440). From the known integral ([10], p. 376, Equation (9.6.19))

$$
I_{v}(z)=\frac{1}{\pi} \int_{0}^{\pi} \mathrm{e}^{z \cos t} \cos (v t) \mathrm{d} t, \quad v \in \mathbb{Z}, z \in \mathbb{C}
$$

we conclude

$$
I_{v}(x) I_{\mu}(y)=\frac{1}{4 \pi^{2}} \int_{-\pi}^{\pi} \int_{-\pi}^{\pi} \mathrm{e}^{x \cos t+y \cos u} \cos (v t) \cos (\mu u) \mathrm{d} t \mathrm{~d} u,
$$

where $v, \mu \in \mathbb{Z}, x, y \in \mathbb{R}$, which will be our starting point. However, in order to get a suitable expression for Equation (9) we rewrite the integrand with the substitution $t+u=2 \theta, t-u=2 \eta$ by means of

$$
\begin{aligned}
& 2 \cos (v(\theta+\eta)) \cos (\mu(\theta-\eta)) \\
&= \cos ((\nu+\mu) \theta) \cos ((\nu-\mu) \eta)+\cos ((\nu-\mu) \theta) \cos ((\nu+\mu) \eta) \\
&-\sin ((\nu+\mu) \theta) \sin ((\nu-\mu) \eta)-\sin ((\nu-\mu) \theta) \sin ((\nu+\mu) \eta) .
\end{aligned}
$$

Moreover, we deduce

$$
\begin{aligned}
x \cos t+y \cos u & =x(\cos t+\cos u)+c \cos u=2 x \cos \theta \cos \eta+c \cos (\theta-\eta) \\
& =(2 x+c) \cos \theta \cos \eta+c \sin \theta \sin \eta=(x+y) \cos \theta \cos \eta+(y-x) \sin \theta \sin \eta .
\end{aligned}
$$


Finally, splitting the integrand into a sum of the odd and even parts we write Equation (9) in the simpler form. The combination of Equations (9) and (10) gives the desired auxiliary integral

$$
\begin{aligned}
I_{\nu}(x) I_{\mu}(y) & =\int_{-\pi}^{\pi} \int_{-\pi}^{\pi} \mathrm{e}^{(x+y) \cos \theta \cos \eta+(y-x) \sin \theta \sin \eta} \cos (\mu+v) \theta \cos (\mu-v) \eta \frac{\mathrm{d} \theta \mathrm{d} \eta}{4 \pi^{2}} \\
& =\frac{1}{4 \pi^{2}} \int_{-\pi}^{\pi}\left\{\int_{-\pi}^{\pi} \mathrm{e}^{(x+y) \cos \theta \cos \eta+(y-x) \sin \theta \sin \eta} \cos (n \eta) \mathrm{d} \eta\right\} \cos (\mu+v) \theta \mathrm{d} \theta \\
& =: \frac{1}{4 \pi^{2}} \int_{-\pi}^{\pi} \mathscr{A}_{n}(\theta) \cos (\mu+v) \theta \mathrm{d} \theta .
\end{aligned}
$$

At this point we recall the trigonometric definition of the Chebyshev function of the first kind which takes the form of a polynomial of degree $n \in \mathbb{N}_{0}([10]$, p. 776, 22.3.15))

$$
T_{n}(z)=\cos (n \arccos z) ;
$$

and it can be extended to the negative integer index Chebyshev polynomials by the convention ([22], p. 1232, Equation (6))

$$
T_{-n}(z)=\cos (-n(\arccos z))=\cos (n \arccos z)=T_{n}(z), \quad n \in \mathbb{N} .
$$

The Chebyshev $T$ polynomial of degree $n$ possesses also the representation ([10], p. 775, 22.3.6)

$$
T_{n}(z)=\frac{n}{2} \sum_{j=0}^{\left[\frac{n}{2}\right]}(-1)^{j} \frac{(n-j-1) !}{j !(n-2 j) !}(2 z)^{n-2 j}, \quad n \in \mathbb{N}_{0} .
$$

Now, we formulate our main result.

Theorem 3. For all $x, y \in \mathbb{R}$ let us denote $w_{\theta}:=w_{\theta}(x, y)=\mathrm{e}^{\mathrm{i} \theta} x+\mathrm{e}^{-\mathrm{i} \theta} y$, and $\mu, v \in \mathbb{Z}$. Then we have

$$
I_{\nu}(x) I_{\mu}(y)=\frac{1}{\pi} \int_{0}^{\pi} \cos (\mu+v) \theta \cdot T_{\mu-v}\left(\left|w_{\theta}\right|^{-1}|x+y||\cos \theta|\right) I_{\mu-v}\left(\left|w_{\theta}\right|\right) \mathrm{d} \theta .
$$

Proof. In order to prove Equation (14), we need the identity

$$
a \sin z+b \cos z=\sqrt{a^{2}+b^{2}} \cos \left(z-\arctan \left(\frac{a}{b}\right)\right), \quad a, b \in \mathbb{R},
$$

by which we transform the inner $\eta$-integral occuring in Equation (12)

$$
\mathscr{A}_{n}(\theta)=\int_{-\pi}^{\pi} \mathrm{e}^{\left|w_{\theta}\right| \cos (\eta-d)} \cos (n \eta) \mathrm{d} \eta
$$

where $\left|w_{\theta}\right|^{2}=x^{2}+y^{2}+2 x y \cos (2 \theta)$ and $d=\arctan \left(\frac{y-x}{x+y} \tan \theta\right)$. The integrand in Equation (16) is a continuous function on the whole integration interval and in the same time $2 \pi$-periodic, therefore its integral over the length of the period is the same everywhere. Consequently, we have

$$
\begin{aligned}
\mathscr{A}_{n}(\theta) & =\int_{-\pi}^{\pi} \mathrm{e}^{\left|w_{\theta}\right| \cos (\eta-d)} \cos (n(\eta-d)+n d) \mathrm{d} \eta=\int_{-\pi}^{\pi} \mathrm{e}^{\left|w_{\theta}\right| \cos \eta} \cos (n \eta+n d) \mathrm{d} \eta \\
& =\cos (n d) \int_{-\pi}^{\pi} \mathrm{e}^{\left|w_{\theta}\right| \cos \eta} \cos (n \eta) \mathrm{d} \eta-\sin (n d) \int_{-\pi}^{\pi} \mathrm{e}^{\left|w_{\theta}\right| \cos \eta} \sin (n \eta) \mathrm{d} \eta .
\end{aligned}
$$

The first integral's integrand is an even function, the second integral's integrand is odd, therefore

$$
\mathscr{A}_{n}(\theta)=2 \pi \cos (n d) I_{n}\left(\left|w_{\theta}\right|\right) \text {. }
$$


Observing the property Equation (13), that is $T_{n}(\cos \theta)=\cos (n \theta)$, we recognize that

$$
\mathscr{A}_{n}(\theta)=2 \pi T_{n}(\cos d) I_{n}\left(\left|w_{\theta}\right|\right) \text {. }
$$

Inserting the last relation into Equation (12) we get

$$
\begin{aligned}
I_{\nu}(x) I_{\mu}(y) & =\frac{1}{2 \pi} \int_{-\pi}^{\pi} \cos ((\mu+v) \theta) T_{n}(\cos d) I_{n}\left(\left|w_{\theta}\right|\right) \mathrm{d} \theta \\
& =\frac{1+(-1)^{n}}{2 \pi} \int_{0}^{\pi} \cos ((\mu+v) \theta) T_{n}(\cos d) I_{n}\left(\left|w_{\theta}\right|\right) \mathrm{d} \theta
\end{aligned}
$$

which is in fact the asserted result, being $\cos d=\left|w_{\theta}\right|^{-1}|x+y||\cos \theta|$. The rest is obvious.

Remark 1. While the elegant expression in Equation (14) contains Chebyshev polynomials, we can re-write equivalently the integrand by using hypergeometric ${ }_{2} F_{1}$, Gegenbauer $C_{\alpha}^{0}$, Kampé de Fériet generalized hypergeometric $F$ function of two variables, Meijer $G_{22}^{12}$ functions among others.

By methodological reasons, not mentioning the related Chebyshev polynomials, we prove in detail the special cases $\mu-v=1,2$, and 3 by virtue of multiple arguments transformations for involved trigonometric functions which are applied during the proof procedure.

Corollary 3. For all $x, y \in \mathbb{R}$ let us denote $w_{\theta}:=w_{\theta}(x, y)=\mathrm{e}^{\mathrm{i} \theta} x+\mathrm{e}^{-\mathrm{i} \theta} y$ and let $\mu, v$ be integers. Then we have

$$
I_{\nu}(x) I_{\mu}(y)= \begin{cases}\frac{|x+y|}{\pi} \int_{0}^{\pi} \frac{|\cos \theta|}{\left|w_{\theta}\right|} \cos (2 v \pm 1) \theta \cdot I_{1}\left(\left|w_{\theta}\right|\right) \mathrm{d} \theta & \mu \mp v=1, \\ \frac{1}{\pi} \int_{0}^{\pi} \frac{2 x y+\left(x^{2}+y^{2}\right) \cos (2 \theta)}{\left|w_{\theta}\right|^{2}} \cos (2 v \pm 2) \theta \cdot I_{2}\left(\left|w_{\theta}\right|\right) \mathrm{d} \theta & \mu \mp v=2, \\ \frac{|x+y|}{\pi} \int_{0}^{\pi} \frac{3 x y \cos \theta+\left(x^{2}-x y+y^{2}\right) \cos (3 \theta)}{\left|w_{\theta}\right|^{3}} & \mu \mp v=3 .\end{cases}
$$

Here, $\operatorname{sgn}(x)$ stands for the sign of a real number $x$.

Proof. Firstly, consider the case $\mu-v=1$. The combination of Equation (16), the series expansion of exponential function, the additional formula for cosine and the binomial formula yields

$$
\mathscr{A}_{1}(\theta)=\sum_{n \geq 0} \frac{\left|w_{\theta}\right|^{n}}{n !} \sum_{k=0}^{n}\left(\begin{array}{l}
n \\
k
\end{array}\right)(\cos d)^{n-k}(\sin d)^{k} \int_{-\pi}^{\pi}(\cos \eta)^{n-k+1}(\sin \eta)^{k} \mathrm{~d} \eta
$$

The involved integral

$$
\mathscr{B}_{k, n}=\int_{-\pi}^{\pi}(\cos \eta)^{n-k+1}(\sin \eta)^{k} \mathrm{~d} \eta=\left(1+(-1)^{k}\right)\left(1+(-1)^{n-k+1}\right) \frac{\Gamma\left(\frac{n-k+2}{2}\right) \Gamma\left(\frac{k+1}{2}\right)}{2 \Gamma\left(\frac{n+3}{2}\right)}
$$

is non-vanishing only for $k$ even and a fortiori $n$ odd. So, the positive solution reads

$$
\mathscr{B}_{2 k, 2 n+1}=\frac{2}{(n+1) !} \Gamma\left(n-k+\frac{3}{2}\right) \Gamma\left(k+\frac{1}{2}\right), \quad k \leq n ; k, n \in \mathbb{N}_{0} .
$$


Accordingly

$$
\begin{aligned}
\mathscr{A}_{1}(\theta) & =\sum_{n \geq 0} \frac{\left|w_{\theta}\right|^{2 n+1}}{(2 n+1) !} \sum_{k=0}^{n}\left(\begin{array}{c}
2 n+1 \\
2 k
\end{array}\right)(\cos d)^{2 n+1-2 k}(\sin d)^{2 k} \mathscr{B}_{2 k, 2 n+1} \\
& =2 \sum_{n \geq 0} \frac{\left(\left|w_{\theta}\right| \cos d\right)^{2 n+1}}{(2 n+1) !(n+1) !} \sum_{k=0}^{n}\left(\begin{array}{c}
2 n+1 \\
2 k
\end{array}\right) \Gamma\left(n-k+\frac{3}{2}\right) \Gamma\left(k+\frac{1}{2}\right)(\tan d)^{2 k} .
\end{aligned}
$$

Now, we transform by the Legendre duplication formula $\sqrt{\pi} \Gamma(2 z)=2^{2 z-1} \Gamma(z) \Gamma\left(z+\frac{1}{2}\right), 2 z \notin$ $\mathbb{Z} \backslash \mathbb{N}$, the binomial coefficient in the sum

$$
\begin{aligned}
\sum_{k=0}^{n}\left(\begin{array}{c}
2 n+1 \\
2 k
\end{array}\right) \Gamma\left(n-k+\frac{3}{2}\right) \Gamma\left(k+\frac{1}{2}\right) a^{2 k} & =\sum_{k=0}^{n} \frac{\Gamma(2 n+2) \Gamma\left(n-k+\frac{3}{2}\right) \Gamma\left(k+\frac{1}{2}\right)}{\Gamma(2 k+1) \Gamma(2 n-2 k+2)} a^{2 k} \\
& =\sqrt{\pi} \Gamma\left(n+\frac{3}{2}\right) \sum_{k=0}^{n}\left(\begin{array}{l}
n \\
k
\end{array}\right) a^{2 k}=\sqrt{\pi} \Gamma\left(n+\frac{3}{2}\right)\left(1+a^{2}\right)^{n}
\end{aligned}
$$

by which, setting $a=\tan d$, Equation (18) becomes

$$
\mathscr{A}_{1}(\theta)=2 \sqrt{\pi} \cos d \sum_{n \geq 0} \frac{\Gamma\left(n+\frac{3}{2}\right)\left|w_{\theta}\right|^{2 n+1}}{(2 n+1) !(n+1) !}=2 \pi \cos d \cdot I_{1}\left(\left|w_{\theta}\right|\right) .
$$

Substituting this expression into Equation (11), we get

$$
\begin{aligned}
I_{\nu}(x) I_{\mu}(y) & =\frac{1}{4 \pi^{2}} \int_{-\pi}^{\pi} \cos (2 v+1) \theta \mathscr{A}_{1}(\theta) \mathrm{d} \theta \\
& =\frac{1}{2 \pi} \int_{-\pi}^{\pi} \cos \left(\arctan \left(\frac{y-x}{x+y} \tan \theta\right)\right) \cos (2 v+1) \theta \cdot I_{1}\left(\left|w_{\theta}\right|\right) \mathrm{d} \theta
\end{aligned}
$$

which is equal to the asserted formula being $\cos (\arctan (z))=1 / \sqrt{1+z^{2}}$.

When $\mu-v=2,3$ by $\cos (2 t)=\cos ^{2} t-\sin ^{2} t$ and $\cos (3 t)=4 \cos ^{3} t-3 \cos t$ we obtain

$$
\begin{aligned}
& \int_{-\pi}^{\pi}(\cos t)^{n-k}(\sin t)^{k} \cos (\mu-v) t \mathrm{~d} t \\
& =\left\{\begin{array}{ll}
\left(1+(-1)^{k}\right)\left(1+(-1)^{n-k}\right)(n-2 k) \frac{\Gamma\left(\frac{n-k+1}{2}\right) \Gamma\left(\frac{k+1}{2}\right)}{4 \Gamma\left(\frac{n+4}{2}\right)}, & \mu-v=2 \\
\left(1+(-1)^{k}\right)\left(1+(-1)^{n-k+1}\right) \frac{n-4 k-1}{2(n+3)} \frac{\Gamma\left(\frac{n-k+2}{2}\right) \Gamma\left(\frac{k+1}{2}\right)}{\Gamma\left(\frac{n+3}{2}\right)}, & \mu-v=3
\end{array} .\right.
\end{aligned}
$$

Hence,

$$
\mathscr{A}_{\mu-v}(\theta)=2 \pi \cos (\mu-v) d \cdot I_{\mu-v}\left(\left|w_{\theta}\right|\right), \quad \mu-v=2,3,
$$

which implies that

$$
I_{\nu}(x) I_{\mu}(y)= \begin{cases}\frac{1}{\pi} \int_{0}^{\pi} \cos (2 d) \cos (2 v+2) \theta \cdot I_{2}\left(\left|w_{\theta}\right|\right) \mathrm{d} \theta, & \mu-v=2 \\ \frac{1}{\pi} \int_{0}^{\pi} \cos (3 d) \cos (2 v+3) \theta \cdot I_{3}\left(\left|w_{\theta}\right|\right) \mathrm{d} \theta, & \mu-v=3\end{cases}
$$


The identities

$$
\cos ((\mu-v) \arctan (z))= \begin{cases}\frac{1-z^{2}}{1+z^{2}} & \mu-v=2 \\ \frac{1-3 z^{2}}{\left(1+z^{2}\right)^{\frac{3}{2}}} & \mu-v=3\end{cases}
$$

complete the proof of Equation (17).

In order to prove the cases $\mu+v=1,2,3$ we point out that now we should solve the integral Equation (11) with respect to $\theta$, that is

$$
\begin{aligned}
\mathscr{C}_{\mu+v}(\eta) & :=\int_{-\pi}^{\pi} \mathrm{e}^{(x+y) \cos \theta \cos \eta+(y-x) \sin \theta \sin \eta} \cos (\mu+v) \theta \mathrm{d} \theta \\
& =\int_{-\pi}^{\pi} \mathrm{e}^{\left|w_{\eta}\right| \cos \left(\eta-d^{\prime}\right)} \cos (\mu+v) \theta \mathrm{d} \theta,
\end{aligned}
$$

where $\left|w_{\eta}\right|^{2}=x^{2}+y^{2}+2 x y \cos (2 \eta)$ and $d^{\prime}=\arctan \left(\frac{y-x}{x+y} \tan \eta\right)$. The rest is obvious.

Remark 2. Three Chebyshev polynomials of the first kind are considered mutatis mutandis in Corollary 3. are $T_{1}(z)=z, T_{2}(z)=2 z^{2}-1$ and $T_{3}(z)=4 z^{3}-3 z$. Namely, for $\mu-v=1$ and $z=\cos d=$ $\left|w_{\theta}\right|^{-1}|x+y||\cos \theta|$ we immediately get the first integral stated in Corollary 3. In a similar way, with the aid of formulae for $T_{2}(z)$ and $T_{3}(z)$ by routine trigonometric transformations we arrive at the remaining two integrals in Equation (17) when $\mu-v=2,3$, respectively.

\section{Discussion: Further Research Goals and Open Problems}

A. The starting tool in the proving process of our main result is the integral Equation (8) which originates back to the ancestor formula ([3], p. 181, Equation (4))

$$
I_{v}(z)=\frac{1}{\pi} \int_{0}^{\pi} \mathrm{e}^{z \cos t} \cos (v t) \mathrm{d} t-\frac{\sin (v \pi)}{\pi} \int_{0}^{\infty} \mathrm{e}^{-z \cosh t-v t} \mathrm{~d} t
$$

which holds true for general $v \in \mathbb{R}$ and complex $z \in \mathbb{C}$. Obviously, for integer order $v=n \in \mathbb{Z}$ this formula takes the form of Equation (8). However, Watson ([3], p. 181) attributed this formula to Schläfli [23], who expressed his result in terms of a confluent hypergeometric function; in turn, this is close to another writing of the Bessel function of the first kind, compare Equation (7).

B. The argument range in Theorem 3 cannot be extended to $\mathbb{C}$ applying the approach of Watson's integrand transformation (see ([3], p. 440)), which is based on the trigonometric Equation (15). Indeed, Equation (15) being valid exclusively in the set of reals, another proving procedure should be used in extending Theorem 3 to complex arguments.

However, it would be of considerable interest to complement the result of Theorem 3 either (i) for general, real orders $v, \mu$ of the input modified Bessel functions of the first kind in $I_{\nu}(x) I_{\mu}(y)$ constrained to have integer sum/difference $\mu \pm v=n \in \mathbb{Z}$, or (ii) to consider the case $\mu \pm v \in \mathbb{R}$. For these tasks the generalized Chebyshev polynomials, and in conjunction with the Gaussian hypergeometric function's special case which turns out to be the cosine function ([19], p. 210, Equation (11)), read

$$
T_{a}(\cos z)=\cos (a z)={ }_{2} F_{1}\left[\begin{array}{c}
\frac{a}{2},-\frac{a}{2} \\
\frac{1}{2}
\end{array} \mid \sin ^{2} z\right], \quad|\sin z|<1,
$$

and could be the appropriate tool. Obviously, these goals overgrow the purposes and tasks of the recent article; we leave it for another address. 
C. A differential operator identity for expressing higher integer order modified Bessel functions in terms of $I_{0}(x)$ is also known and reads as follows

$$
I_{n}(x)=T_{n}\left(D_{x}\right) I_{0}(x), \quad D_{x}:=\frac{\mathrm{d}}{\mathrm{d} x} .
$$

Applying this identity to Equation (14) in Theorem 3, we conclude

$$
I_{v}(x) I_{\mu}(y)=\left.\frac{1}{\pi} \int_{0}^{\pi} \cos (\mu+v) \theta \cdot T_{\mu-v}\left(\left|w_{\theta}\right|^{-1}|x+y||\cos \theta|\right) T_{\mu-v}\left(D_{t}\right) I_{0}(t)\right|_{t=\left|w_{\theta}\right|} \mathrm{d} \theta .
$$

D. The need for efficient functional or uniform bounding inequalities for Bessel-type special functions frequently occur in convergence issues regarding the so-called Fourier-Bessel series of the Neumann, Kapteyn, Schlömilch and Dini kind, see [16,24]. However, in applications conditioned with numerical approximations which occur in numerical modeling in the STEM disciplines, almost all Bessel and alike functions' arguments are real, consult B. The new integral Equation (14) of the product of two Bessel and/or modified Bessel functions can be adequate for establishing new bounding inequalities.

E. Finally, we have to say a few words concerning the interrelations between the integral expressions exposed in Theorems 1-3.

In Theorems 1 and 2 we present three different integral representations for the product of two Bessel functions of the first kind. It can be shown that the first Equation (3) in Theorem 1 and Equation (5) of Theorem 2 can coincide by suitably chosen involved parameters. Indeed, setting $\mu=v$ in Equation (3) we have ([19], p. 216, Equation (41))

$$
J_{v}^{2}(x)=\frac{x^{2 v}}{2^{2 v} \Gamma^{2}(v+1)}{ }_{1} F_{2}\left[\begin{array}{c}
v+\frac{1}{2} \\
v+1,2 v+1
\end{array} \mid-x^{2}\right]=: R_{1} .
$$

On the other hand for $a=b=1$ and applying the substitutions $u=t-1$ and $y=t / 2$, respectively, Equation (5) becomes the integral

$$
J_{v}^{2}(x)=\frac{x^{v}}{\sqrt{\pi} \Gamma\left(v+\frac{1}{2}\right)} \int_{0}^{1}(1-y)^{v-1 / 2} y^{(v-1) / 2} J_{v}(2 x \sqrt{y}) \mathrm{d} y=: R_{2} .
$$

In turn, the power series definition of the Bessel function in the integrand and the term-wise integration transform the right-hand side expression into

$$
R_{2}=\frac{x^{v}}{\sqrt{\pi} \Gamma\left(v+\frac{1}{2}\right)} \sum_{n \geq 0} \frac{\left(-x^{2}\right)^{n}}{\Gamma(v+n+1) n !} \mathrm{B}\left(n+v+\frac{1}{2}, v+\frac{1}{2}\right),
$$

which, after some routine calculation takes the form $R_{1}$.

F. While we easily transform the results presented in Theorems 1 and 2 using $J_{v}(\mathrm{i} x)=\mathrm{i}^{v} I_{v}(x)$, it is important to mention that it is not possible to do the same with Theorem 3 and the reversed connection formula $I_{v}(\mathrm{i} x)=\mathrm{i}^{v} J_{v}(x)$, because Theorem 3 is valid only for real arguments (consult B.)

Author Contributions: conceptualization, D.J.M. and T.K.P.; methodology, D.J.M. and T.K.P.; validation D.J.M. and T.K.P.; formal analysis, D.J.M. and T.K.P.; writing-Original draft preparation, D.J.M. and T.K.P.; writing-Review and editing, D.J.M. and T.K.P.

Funding: This research received no external funding.

Acknowledgments: Both authors are indebted to all four unknown referees and to the editor for several constructive comments which mainly improve the exposition's relevance and completeness and finally encompass the article.

Conflicts of Interest: The authors declares no conflict of interest. 


\section{References}

1. Schläfli, L. Einige Bemerkungen zu Herrn Neumann's Untersuchungen über die Bessel'schen Funktionen. Math. Ann. 1871, III, 134-149.

2. Gegenbauer, L. Über eine Relation des Herrn Hobson. Wien. Sitzungsberichte 1902, CXI, 563-572.

3. Watson, G.N. A Treatise on the Theory of Bessel Functions; Cambridge University Press: London, UK, 1922.

4. Dixon, A.L.; Ferrar, W.L. Integrals for the product of two Bessel functions. Q. J. Math. Oxf. Ser. 1933, 4, 193-208. [CrossRef]

5. Nicholson, J.W. The asymptotic expansions of Bessel functions. Phil. Mag. 1910, 19, 228-249. [CrossRef]

6. Dixon, A.L.; Ferrar, W.L. Infinite integrals in the theory of Bessel functions. Q. J. Math. Oxf. Ser. 1930, 1, 122-145. [CrossRef]

7. Görlich, E.; Markett, C.; Stüpp, O. Integral formulas associated with products of Bessel functions: A new partial differential equation approach. J. Comput. Appl. Math. 1994, 51, 135-157. [CrossRef]

8. Glasser, M.L.; Montaldi, E. Some integrals involving Bessel functions. J. Math. Anal. Appl. 1994, 183, 577-590. [CrossRef]

9. Hrycak, T.; Schmutzhard, S. A Nicholson-type integral for the cross-product of the Bessel functions. J. Math. Anal. Appl. 2016, 436, 168-178. [CrossRef]

10. Abramowitz, M.; Stegun, I.A. (Eds.) Handbook of Mathematical Functions with Formulas, Graphs, and Mathematical Tables; Applied Mathematics Series 55, National Bureau of Standards, Washington, DC, USA, 1964, 9th Reprinted Edition; Dover Publications: New York, NY, USA, 1972.

11. Erdélyi, A.; Magnus, W.; Oberhettinger, F.; Tricomi, F.G. Higher Transcendental Functions; Based, In Part, on Notes Left by Harry Bateman; McGraw-Hill Book Company, Inc.: New York, NY, USA; Toronto, UK; London, UK, 1953; Volume II.

12. Gradshteyn, I.S.; Ryzhik, I.M. Table of Integrals, Series, and Products, 6th ed.; Translated from the Russian. Translation Edited and with a preface by Alan Jeffrey and Daniel Zwillinger; Academic Press, Inc.: San Diego, CA, USA, 2000.

13. Luke, Y.L. Integrals of Bessel Functions; McGraw-Hill Book Co., Inc.: New York, NY, USA; Toronto, UK; London, UK, 1962.

14. Magnus, W.; Oberhettinger, F. Formeln und Sütze für die speziellen Funktionen der mathematischen Physik, 2nd ed.; Springer: Berlin, Germany, 1948.

15. Olver, F.W.J.; Lozier, D.W.; Boisvert, R.F.; Clark, C.W. (Eds.) NIST Handbook of Mathematical Functions; NIST and Cambridge University Press: Cambridge, UK, 2010.

16. Baricz, Á.; Jankov Maširević, D.; Pogány, T.K. Series of Bessel and Kummer-Type Functions; Lecture Notes in Mathematics, 2207; Springer: Cham, Switzerland, 2017.

17. Simon, B. Harmonic Analysis: A Comprehensive Course in Analysis; Part 3; American Mathematical Society: Providence, RI, USA, 2015.

18. Vilenkin, N.Y. Special Functions and the Theory of Group Representations; Translated from the Russian by V. N. Singh. Translations of Mathematical Monographs, Vol. 22; American Mathematical Society: Providence, RI, USA, 1968.

19. Luke, Y.L. The Special Functions and Their Approximations; Mathematics in Science and Engineering Vol. 53: Academic Press: New York, NY, USA; London, UK, 1969; Volume I.

20. Erdélyi, A.; Magnus, W.; Oberhettinger, F.; Tricomi, F.G. Higher Transcendental Functions; Based, in part, on notes left by Harry Bateman; McGraw-Hill Book Company, Inc.: New York, NY, USA; Toronto, UK; London, UK, 1953; Volume I.

21. Gauss Hypergeometric Function 2F1: Specific Values (formula 07.23.03.0171). Available online: http:/ / functions.wolfram.com/HypergeometricFunctions/Hypergeometric2F1/03/06/06/0001/ (accessed on October 13 2019).

22. Rayes, M.O.; Trevisan, V.; Wang, P.S. Factorization properties of Chebyshev polynomials. Comput. Math. Appl. 2005, 50, 1231-1240. [CrossRef] 
23. Schläfli, L. Sopra un teorema di Jacobi recato a forma piu generale ed applicata alia funzione cilindrica. Ann. di Mat. 1873, 1871, 199-205.

24. Baricz, Á.; Pogány, T.K. Properties of the product of modified Bessel functions. In Analytic Number Theory, Approximation Theory, and Special Functions_-In Honor of Hari M. Srivastava; Milovanović, G.V., Rassias, M., Eds.; Springer: New York, NY, USA, 2014; pp. 809-820.

(C) 2019 by the authors. Licensee MDPI, Basel, Switzerland. This article is an open access article distributed under the terms and conditions of the Creative Commons Attribution (CC BY) license (http:/ / creativecommons.org/licenses/by/4.0/). 